\title{
Remarks on continuously distributed sequences
}

\section{Paštéka M.}

In the first part of the paper we define the notion of the density as certain type of finitely additive probability measure and the distribution function of sequences with respect to the density. Then we derive some simple criterions providing the continuity of the distribution function of given sequence. These criterions we apply to the van der Corput's sequences. The Weyl's type criterions of continuity of the distribution function are proven.

Key words and phrases: uniform distribution, density, van der Corput's sequence.

Department of Mathematics and Informatics, Faculty of Education, University of Trnava, Priemyselna 4, Trnava, Slovakia E-mail: pasteka@mat.savba.sk

\section{Introduction}

The aim of this paper is to study the distribution properties of sequences of real numbers. The research of distribution of sequences connected with certain type of measure of sets of their indexes was started by Herman Weyl in his famous paper [13] in 1916. Let $\mathbb{N}$ be the set of positive integers. For $S \subset \mathbb{N}$ the value

$$
\limsup _{N \rightarrow \infty} \frac{|S \cap[1, N]|}{N}=\bar{d}(S)
$$

is called the upper asymptotic density. We denote

$$
\mathcal{D}=\{S \subset \mathbb{N}: \bar{d}(S)+\bar{d}(\mathbb{N} \backslash S)=1\}
$$

It is easy to check that $\mathcal{D}$ is the system of all sets $S \subset \mathbb{N}$ such that the term on left hand side in (1) has proper limit. In this case its value is called the asymptotic density of $S$, denoted as $d(S)$.

Let $\{v(n)\}$ be a sequence. For an arbitrary set $A$ we denote

$$
v^{-1}(A)=\{n \in \mathbb{N}: v(n) \in A\} .
$$

A sequence of elements of interval $[0,1]$ is called uniformly distributed if and only if for each subinterval $I \subset[0,1]$ we have $v^{-1}(I) \in \mathcal{D}$ and $d\left(v^{-1}(I)\right)=|I|$.

This approach was later generalized by I. Schoenberg in [11]. We say that a sequence $\{v(n)\}$ of elements of interval $[0,1]$ has asymptotic distribution function if and only if for each $x \in[0,1]$ the set $v^{-1}([0, x))$ belongs to $\mathcal{D}$ and in this case the function

$$
F(x)=d\left(v^{-1}([0, x))\right)
$$

is called the asymptotic distribution function of $\{v(n)\}$. 
Our scope is to derive some results for more general concept of density - certain type of finite additive probability measure defined on $q$-algebra or algebra of subsets of $\mathbb{N}$. In the theory of distribution of sequences the question of the continuity of the distribution function plays very important role. In the following text we shall study the sequences from this point of view.

\section{Density}

A system $\mathcal{Y}$ of subsets of $\mathbb{N}$ will be called q-algebra if and only if

i) $\varnothing, \mathbb{N} \in \mathcal{Y}$,

ii) $A \cap B=\varnothing \Longrightarrow A \cup B \in \mathcal{Y}$ for all $A, B \in \mathcal{Y}$,

iii) $\mathbb{N} \backslash A \in \mathcal{Y}$ for all $A \in \mathcal{Y}$.

A finitely additive probability measure $v$ defined on $q$-algebra $\mathcal{Y}$ will be called density if and only if

$$
A \in \mathcal{Y} \Longleftrightarrow \forall \varepsilon>0 \exists A_{1}, A_{2} \in \mathcal{Y}: A_{1} \subset A \subset A_{2} \wedge v\left(A_{2}\right)-v\left(A_{1}\right)<\varepsilon \quad \forall A \subset \mathbb{N} .
$$

Let $v$ be a density defined on $q$-algebra $\mathcal{Y}$. We say that a sequence $\{v(n)\}$ of real numbers is $v$-measurable if and only if for each real number $x$ the set $v^{-1}((-\infty, x))$ belongs to $\mathcal{Y}$. In this case the function $G(x)=v\left(v^{-1}((-\infty, x))\right)$ will be called $v$-distribution function of $\{v(n)\}$. If $G$ is continuous, we say that $\{v(n)\}$ is $v$-continuously distributed.

Theorem 1. A bounded sequence $\{v(n)\}$ of real numbers is $v$-continuously distributed if and only if for some set $S$, which is dense in the real line, we have $v^{-1}((-\infty, x)) \in \mathcal{Y}$ for each $x \in S$ and the function $F(x)=v\left(v^{-1}((-\infty, x))\right)$ is uniformly continuous on $S$. In this case the $v$-distribution function of $\{v(n)\}$ is the unique extension of $F$ to whole real line.

Proof. Let $t$ be an arbitrary real number. For given $\delta>0$ there exist $x_{1}, x_{2} \in S$ such that $x_{1}<t<x_{2}$ and $x_{2}-x_{1}<\delta$. Then

$$
v^{-1}\left(\left(-\infty, x_{1}\right)\right) \subset v^{-1}((-\infty, t)) \subset v^{-1}\left(\left(-\infty, x_{2}\right)\right) .
$$

Since $F$ is uniformly continuous on $S$, the value $\delta>0$ in such manner can be determined such that $F\left(x_{2}\right)-F\left(x_{1}\right)<\varepsilon$. And so, from (2) and (3) we get $v^{-1}((-\infty, t)) \in \mathcal{Y}$. Analogously we can prove that the function $v\left(v^{-1}((-\infty, t))\right)$ is a continuous extension of $F(t)$.

The definition of asymptotic density via upper density can be generalized to the axiomatic form as follows. We say that a set function $\mathbf{n}: P(\mathbb{N}) \rightarrow[0,1]$ is normalized if and only if

$$
\mathbf{n}(\varnothing)=0, \quad \mathbf{n}(\mathbb{N})=1
$$

We say that $\mathbf{n}$ is isotonic if and only if

$$
A \subset B \Longrightarrow \mathbf{n}(A) \leq \mathbf{n}(B) .
$$

If $\mathbf{n}: P(\mathbb{N}) \rightarrow[0,1]$ is isotonic and normalized and for every $A, B \subset \mathbb{N}$ we have

$$
\begin{gathered}
A \cap B=\varnothing \Longrightarrow \mathbf{n}(A \cup B) \leq \mathbf{n}(A)+\mathbf{n}(B), \\
A \cup B=\mathbb{N} \Longrightarrow 1+\mathbf{n}(A \cap B) \leq \mathbf{n}(A)+\mathbf{n}(B),
\end{gathered}
$$

we say that $\mathbf{n}$ is outer density. 
By the standard methods can be proved the following assertion.

Theorem 2. If $\mathbf{n}$ is an outer density then the set system

$$
\mathcal{Y}=\{A \subset \mathbb{N}: \mathbf{n}(A)+\mathbf{n}(\mathbb{N} \backslash A)=1\}
$$

forms a $q$-algebra and $v=\mathbf{n} \mid \mathcal{Y}$ is a density on $\mathcal{Y}$.

In this case we say that $v$ is given by $\mathbf{n}$.

The axiomatic approach of density is studied in the paper [6], inspired also by the work [4] (see also [10]).

From Theorem 1 we get immediately the following result.

Theorem 3. Let $v$ be a density given by $\mathbf{n}$. A sequence $\{v(n)\}$ of real numbers is $v$-continuously distributed if and only if for a suitable set $S$, which is dense in the real line, we have that $v^{-1}((-\infty, x)) \in \mathcal{Y}$ for every $x \in S$ and for each real number $t$

$$
\lim _{\varepsilon \rightarrow 0^{+}} \mathbf{n}\left(v^{-1}((t-\varepsilon, t+\varepsilon))\right)=0
$$

holds. In this case the $v$-distribution function of $\{v(n)\}$ is the unique extension of the function $F(x)=v\left(v^{-1}((-\infty, x))\right), x \in S$, to whole real line.

\section{Application to the van der Corput sequence}

We recall that a $q$-algebra $\mathcal{Y}$ fulfilling the condition

$$
A, B \in \mathcal{Y} \Longrightarrow A \cup B \in \mathcal{Y}
$$

is called algebra. In 1946, R.C. Buck defined Buck's measure density starting from the asymptotic density of arithmetic progressions. Denote $r+(m)=\{n \in \mathbb{N}: n \equiv r(\bmod m)\}$ for $m \in \mathbb{N}, r \in \mathbb{N} \cup\{0\}$. Let $\mathcal{D}_{0}$ be the algebra of all sets of the form $\bigcup_{i=1}^{s} r_{i}+\left(m_{i}\right)$, where $m_{i} \in \mathbb{N}$ and $r_{i} \in \mathbb{N} \cup\{0\}$. Clearly $\mathcal{D}_{0} \subset \mathcal{D}$. The set function $\mu^{*}$ is defined by

$$
\mu^{*}(S)=\inf \left\{d(A): A \in \mathcal{D}_{0} \wedge S \subset A\right\}
$$

for all $S \subset \mathbb{N}$ is called Buck's measure density. Clearly $\mu^{*}$ is isotonic and normalized. Moreover, the inequality

$$
\mu^{*}(A \cup B)+\mu^{*}(A \cap B) \leq \mu^{*}(A)+\mu^{*}(B)
$$

holds for $A, B \subset \mathbb{N}$. Thus, the set system

$$
\mathcal{D}_{\mu}=\left\{A \subset \mathbb{N}: \mu^{*}(A)+\mu^{*}(\mathbb{N} \backslash A)=1\right\}
$$

is an algebra of sets and the restriction $\mu=\left.\mu^{*}\right|_{\mathcal{D}_{\mu}}$ is density on $\mathcal{D}_{\mu}$. Clearly $r+(m) \in \mathcal{D}_{\mu}$ and

$$
\mu(r+(m))=\frac{1}{m}
$$

for $m \in \mathbb{N}$ and $r \in \mathbb{N} \cup\{0\}$. For the details we refer to $[1,8,9]$. 
Theorem 3 implies the following assertion.

Corollary 1. A sequence $\{v(n)\}$ of elements of unit interval has a continuous $v$ distribution function if and only if there exists a system $S_{n}=\left\{0=x_{0}^{(n)}<x_{1}<\cdots<x_{k_{n}}^{(n)}=1\right\}, n \in \mathbb{N}$, of finite sequences of elements of the unit interval such that $\lim _{n \rightarrow \infty}\left(x_{k+1}^{(n)}-x_{k}^{(n)}\right)=0$ uniformly for $k=0, \ldots, k_{n}-1$, the sets $v^{-1}\left(\left[x_{k}^{(n)}, x_{k+1}^{(n)}\right]\right)$ belong to $\mathcal{Y}$ and $\lim _{n \rightarrow \infty} v\left(v^{-1}\left(\left[x_{k}^{(n)}, x_{k+1}^{(n)}\right]\right)\right)=0$ uniformly for $k=0, \ldots, k_{n}-1$.

The following sequence was studied firstly by van der Corput (see $[3,5,12])$. Let $\left\{B_{n}\right\}$ be an increasing sequence of positive integers such that $B_{n} \mid B_{n+1}, n \in \mathbb{N}$. Every positive integer $a$ can be uniquely written in the form

$$
a=c_{0}+c_{1} B_{1}+\cdots+c_{j} B_{j}
$$

where $0 \leq c_{0}<B_{1}, 0 \leq c_{i}<\frac{B_{i}}{B_{i-1}}, i=2, \ldots, j$. This allows us associate to this $a$ the value

$$
v(a)=\frac{c_{0}}{B_{1}}+\cdots+\frac{c_{j}}{B_{j+1}} .
$$

The following one to one correspondence between the subintervals of $[0,1)$ and the arithmetic progressions plays an important role. For each subinterval $\left[\frac{j-1}{B_{k}}, \frac{j}{B_{k}}\right), 1 \leq j \leq B_{k}, k \in \mathbb{N}$, there exists $\ell_{j}^{(k)} \in\left\{0,1, \ldots, B_{k}-1\right\}$ such that

$$
n \in \ell_{j}^{(k)}+\left(B_{k}\right) \Longleftrightarrow v(n) \in\left[\frac{j-1}{B_{k}}, \frac{j}{B_{k}}\right) .
$$

A sequence $\{h(n)\}$ of positive integers is called Buck uniformly distributed in $\mathbb{Z}$ if and only if $h^{-1}(r+(m)) \in \mathcal{D}_{\mu}$ and $\mu\left(h^{-1}(r+(m))\right)=\frac{1}{m}$ for each $m \in \mathbb{N}$ and $r \in \mathbb{N} \cup\{0\}$.

Theorem 4. Let $\left\{B_{k}\right\}$ fulfils the following condition: for all $m \in \mathbb{N}$ there exists $k$ such that $m \mid B_{k}$. Then for every sequence $\left\{k_{n}\right\}$ of positive integers the sequence $\left\{v\left(k_{n}\right)\right\}$ is Buck uniformly distributed if and only if the sequence $\left\{k_{n}\right\}$ is Buck uniformly distributed in $\mathbb{Z}$.

Theorem 5. Let $g: \mathbb{N} \rightarrow \mathbb{N}$ be such mapping that the condition

$$
g\left(x_{1}\right) \equiv g\left(x_{2}\right) \quad\left(\bmod B_{k}\right) \quad \Longleftrightarrow \quad x_{1} \equiv x_{2} \quad\left(\bmod B_{k}\right)
$$

holds for every $x_{1}, x_{2} \in \mathbb{N}, k=1,2,3, \ldots$ Then the sequence $\{v(g(n))\}$ is Buck uniformly distributed.

Proof. Put $I_{j}^{(k)}=\left[\frac{j-1}{B_{k}}, \frac{j}{B_{k}}\right)$. Then $x \in v^{-1}\left(I_{j}^{(k)}\right)$ if and only if $g(x) \in \ell_{j}^{(k)}+\left(B_{k}\right)$, for $x, k \in \mathbb{N}$, $0 \leq j<B_{k}$. The values $g(1), \ldots, g\left(B_{k}\right)$ are incongruent modulo $B_{k}, k \in \mathbb{N}$, and so there exist $r_{j}^{(k)}$ such that $g\left(r_{j}^{(k)}\right) \equiv \ell_{j}^{(k)}\left(\bmod B_{k}\right)$. Put $u(n)=v(g(n)), n \in \mathbb{N}$. Then

$$
u^{-1}\left(I_{j}^{(k)}\right)=r_{j}^{(k)}+\left(B_{k}\right)
$$

and so $u^{-1}\left(I_{j}^{(k)}\right) \in \mathcal{D}_{\mu}$ and $\mu\left(u^{-1}\left(I_{j}^{(k)}\right)\right)=\frac{1}{B_{k}}$. The end points of intervals $I_{j}^{(k)}, j=1, \ldots, B_{k}$, $k \in \mathbb{N}$, form a dense subset in $[0,1]$ and the assertion follows. 
Corollary 2. If $m \in \mathbb{N}$ is such that $\left(m, B_{k}\right)=1, k=1,2,3, \ldots$, then the sequence $\{v(m n)\}$ is Buck uniformly distributed.

Corollary 3. Let $s \in \mathbb{N}$ and $p_{j}, j=1,2,3, \ldots$, be a sequence of different primes such that $\left(s, p_{j}-1\right)=1, j=1,2,3, \ldots$ If $B_{k}=p_{1} \ldots p_{k}, j=1,2,3, \ldots$, then the sequence $\left\{v\left(n^{s}\right)\right\}$ is Buck uniformly distributed.

Theorem 6. Let $p>2$ be a prime number. Suppose that $r \in \mathbb{N}$ is a positive integer which is primitive root modulo $p^{\alpha}, \alpha=1,2,3, \ldots$ If $B_{k}=p^{k}, k=1,2,3, \ldots$, then the sequence $\left\{v\left(r^{n}\right)\right\}$ is Buck measurable and the function

$$
F(x)=\left\{\begin{array}{cl}
0, & x \leq \frac{1}{p} \\
\frac{p x-1}{p-1}, & x \in\left(\frac{1}{p}, 1\right],
\end{array}\right.
$$

is its Buck's distribution function.

Proof. Put $u(n)=v\left(r^{n}\right), n \in \mathbb{N}$. The numbers $r^{n}, n \in \mathbb{N}$, are relatively prime with $p$. Thus if

$$
r^{n}=c_{0}+c_{1} p+\cdots+c_{j} p^{j}
$$

then $c_{0}>0$ and so $v\left(r^{n}\right) \geq \frac{1}{p}, n \in \mathbb{N}$. This yields that $u^{-1}\left(\left(0, \frac{1}{p}\right)\right)=\varnothing$. Suppose $\alpha>0$. For every $s \in \mathbb{N},(s, p)=1$, there exists a unique $n_{s} \in\left\{0, \ldots, \varphi\left(p^{\alpha}\right)-1\right\}$ such that

$$
n \in n_{s}+\left(\varphi\left(p^{\alpha}\right)\right) \Longleftrightarrow r^{n} \in s+\left(p^{\alpha}\right) \text {. }
$$

This implies

$$
u^{-1}\left(\left[\frac{b}{p^{\alpha}}, \frac{b+1}{p^{\alpha}}\right)\right)=\ell_{b}+\left(\varphi\left(p^{\alpha}\right)\right), \quad b=p^{\alpha-1}, \ldots, p^{\alpha}-1,
$$

for suitable $\ell_{b}$. Therefore

$$
\mu\left(u^{-1}\left(\left[\frac{b}{p^{\alpha}}, \frac{b+1}{p^{\alpha}}\right)\right)\right)=\frac{1}{p\left(p^{\alpha-1}-1\right)} .
$$

And so a simple calculation gives

$$
\mu\left(u^{-1}[0, x)\right)=F(x)
$$

for $x \in S=\left\{\frac{a}{p^{\alpha}}: a=0, \ldots, p^{\alpha}, \alpha \in \mathbb{N}\right\}$. This set $S$ is dense in the unit interval and the assertion follows.

Theorem 7. Let $p_{j}, j=1,2,3, \ldots$, be pairwise distinct prime numbers. Put $B_{k}=p_{1} \ldots p_{k}$, $k \in \mathbb{N}$. If $f(x)$ is a polynomial of degree greater than 0 with positive integer coefficients, then the sequence $\{v(f(n))\}$ is Buck measurable and its Buck's distribution function is continuous.

Proof. For every $k \in \mathbb{N}, r=0, \ldots, B_{k}-1$, we have

$$
f(x) \in r+\left(B_{k}\right) \Longleftrightarrow f(x) \equiv r \quad\left(\bmod p_{j}\right), \quad j=1, \ldots, k .
$$

Due to the Chinese reminder theorem this system of congruences has at most $d^{k}$ solutions, where $d$ is degree of $f(x)$. From this we can conclude that for the sequence $\{u(n)\}=\{v(f(n))\}$ the set $u^{-1}\left(\left[\frac{j}{B_{k}}, \frac{j+1}{B_{k}}\right)\right)$ is union at most $d^{k}$ arithmetic progression with modul $B_{k}$. Therefore it is Buck measurable and

$$
\lim _{k \rightarrow \infty} \mu\left(u^{-1}\left(\left[\frac{j}{B_{k}}, \frac{j+1}{B_{k}}\right)\right)\right) \leq \lim _{k \rightarrow \infty} \frac{d^{k}}{B_{k}}=0 .
$$




\section{Matrix density}

We can apply these statements for the matrix density studied originally in the paper [2], later in [4] and other papers.

Proposition 1. Let $\mathbf{n}_{k}, k=1,2,3, \ldots$, be probability measures on $P(\mathbb{N})$. Then the set function

$$
\mathbf{n}(A)=\limsup _{k \rightarrow \infty} \mathbf{n}_{k}(A)
$$

is an outer density.

Thus the set system

$$
\mathcal{Y}=\{A \subset \mathbb{N}: \mathbf{n}(A)+\mathbf{n}(\mathbb{N} \backslash A)=1\}
$$

is a $q$-algebra and $v=\mathbf{n} \mid \mathcal{Y}$ is a density.

As ussualy, we denote by $\mathcal{X}_{S}$ the indicator function of the set $S$.

Proposition 2. For $A \subset \mathbb{N}$ we have

$$
A \in \mathcal{Y} \Longleftrightarrow \lim _{k \rightarrow \infty} \sum_{n=1}^{\infty} \mathbf{n}_{k}(\{n\}) \mathcal{X}_{A}(n)=\mathbf{n}(A)
$$

For a sequence $\{v(n)\}$ of real numbers and an arbitrary interval $I$ we have

$$
\mathcal{X}_{v^{-1}(I)}(n)=\mathcal{X}_{I}(v(n)) \text {. }
$$

This equation will be useful for the proof of the following result.

Theorem 8. A sequence $\{v(n)\}$ of elements of $[0,1]$ is $v$-continuously distributed if and only if for each real function $f$, which is continuous on $[0,1]$,

a) the limit

$$
\lim _{k \rightarrow \infty} \sum_{n=1}^{\infty} f(v(n)) \mathbf{n}_{k}(\{n\})
$$

exists and

b) for every $x \in[0,1]$ we have

$$
\lim _{\varepsilon \rightarrow 0^{+}} \limsup _{k \rightarrow \infty} \sum_{n=1,|v(n)-x|<\varepsilon}^{\infty}|f(v(n))| \mathbf{n}_{k}(\{n\})=0 .
$$

Proof. One implication is trivial.

Using Riesz representation theorem, we can prove by standard procedure that

$$
\lim _{k \rightarrow \infty} \sum_{n=1}^{\infty} f(v(n)) \mathbf{n}_{k}(\{n\})=\int_{0}^{1} f(x) d g(x)
$$

for suitable nondecreasing function $g$, defined on the interval $[0,1]$ such that $g(0)=0$, $g(1)=1$. From Proposition 2 and equality (4) we can derive by standard way that for each point $x$ of continuity of $g$ we have $v^{-1}([0, x)) \in \mathcal{Y}$ and

$$
v\left(v^{-1}([0, x))\right)=g(x) \text {. }
$$

Since the set of points of discontinuity of $g$ is countable, the set of points of continuity of $g$ is dense in $[0,1]$.

Applying the condition $b$ ) to the function $f(x)=1, x \in[0,1]$, we get

$$
\lim _{\varepsilon \rightarrow 0^{+}} \mathbf{n}\left(v^{-1}((x-\varepsilon, x+\varepsilon))\right)=0
$$

for each $x \in[0,1]$ and from Theorem 1 we obtain the assertion. 
For a bounded sequence $\{v(n)\}$ of real numbers, it can be defined the supremum norm as usually

$$
\|v\|=\sup \{|v(n)|: n \in \mathbb{N}\} .
$$

Theorem 8 implies the following assertion.

Corollary 4. The set of all $v$-continuously distributed sequences of elements of $[0,1]$ is closed with respect to the supremum norm.

\section{Measure density}

The Buck's measure density was later generalized in [7].

Let $\mathcal{E}_{n}=\left\{E_{i}^{(n)}: i=1, \ldots, k_{n}\right\}, n \in \mathbb{N}$, be a system of decompositions of $\mathbb{N}$ fulfilling the condition

$$
\begin{aligned}
& \text { for each } n=1,2,3, \ldots, \text { there exists } m \in \mathbb{N} \text { such that } \\
& \text { each set } \mathcal{E}_{j}, 1 \leq j \leq n, \text { is a union of sets } \mathcal{E}_{k} \text { for } k \geq m \text {. }
\end{aligned}
$$

Denote by $\mathcal{A}$ the algebra of all finite unions of sets from $\bigcup_{n=1}^{\infty} \mathcal{E}_{n}$. Suppose that $\Delta$ is a finitely additive probability measure on $\mathcal{A}$. Let us define

$$
\delta^{*}(S)=\inf \{\Delta(A): S \subset A, A \in \mathcal{A}\} .
$$

Immediately we get the following assertion.

Lemma 1. The set function $\delta^{*}$ given by (6) is normalized and isotonic, moreover, for every $S_{1}, S_{2}$ we have

$$
\delta^{*}\left(S_{1} \cup S_{2}\right)+\delta^{*}\left(S_{1} \cap S_{2}\right) \leq \delta^{*}\left(S_{1}\right)+\delta^{*}\left(S_{2}\right)
$$

Denote

$$
\mathcal{D}_{\delta}=\left\{S \subset \mathbb{N}: \delta^{*}(S)+\delta^{*}(\mathbb{N} \backslash S)=1\right\} .
$$

Lemma 1 implies the following assertion.

Proposition 3. The system of sets $\mathcal{D}_{\delta}$ is an algebra of sets and the set function $\delta=\left.\delta^{*}\right|_{\mathcal{D}_{\delta}}$ is a density on $\mathcal{D}_{\delta}$.

We say that a finite sequence $a_{1}, \ldots, a_{j}$ of positive integers is independent with respect to $\mathcal{E}_{n}$, $n \in \mathbb{N}$, if and only if $a_{i_{1}}, a_{i_{2}}$ belong to distinct sets from $\mathcal{E}_{n}$ if $i_{1} \neq i_{2}$.

Let $S \subset \mathbb{N}$. Suppose that for given $n \in \mathbb{N}$ there is the maximal sequence $s_{1}, \ldots s_{k}$ of elements of $S$, independent with respect to $\mathcal{E}_{n}$, such that $s_{j} \in E_{i_{j}}^{(n)}$. Put $H\left(S: \mathcal{E}_{n}\right)=\sum_{j=1}^{k} \Delta\left(E_{i_{j}}^{(n)}\right)$. Using the property (5), the following proposition can be proved by standard procedure (see $[8$, Theorem 5]).

Proposition 4. For each $S \subset \mathbb{N}$ we have

$$
\delta^{*}(S)=\lim _{n \rightarrow \infty} H\left(S: \mathcal{E}_{n}\right)
$$

A finite sequence $\left\{a_{j}^{(n)}, j=1, \ldots, k_{n}\right\}$ of positive integers, such that $a_{j}^{(n)} \in E_{j}^{(n)}, j=1, \ldots, k_{n}$, will be called the representative system of $\mathcal{E}_{n}$.

It is easy to observe the following two facts. 
Proposition 5. Let $\left\{a_{1}^{(n)}, \ldots, a_{k_{n}}^{(n)}\right\}$ be a representative system for $\mathcal{E}_{n}, n \in \mathbb{N}$. Then for every $S \subset \mathbb{N}$ we have

$$
1-H\left(\mathbb{N} \backslash S: \mathcal{E}_{n}\right) \leq \sum_{j=1}^{k_{n}} \mathcal{X}_{S}\left(a_{j}^{(n)}\right) \Delta\left(E_{j}^{(n)}\right) \leq H\left(S: \mathcal{E}_{n}\right) .
$$

Proposition 6. For every $S \subset \mathbb{N}$ there exist representative systems $\left\{a_{1}^{(n)}, \ldots, a_{k_{n}}^{(n)}\right\}$ and $\left\{b_{1}^{(n)}, \ldots, b_{k_{n}}^{(n)}\right\}$ of $\mathcal{E}_{n}$ such that

$$
\lim _{n \rightarrow \infty} \sum_{j=1}^{k_{n}} \mathcal{X}_{S}\left(a_{j}^{(n)}\right) \Delta\left(E_{j}^{(n)}\right)=\delta^{*}(S)
$$

and

$$
\lim _{n \rightarrow \infty} \sum_{j=1}^{k_{n}} \mathcal{X}_{S}\left(b_{j}^{(n)}\right) \Delta\left(E_{j}^{(n)}\right)=1-\delta^{*}(\mathbb{N} \backslash S) .
$$

And so, we get the following result.

Lemma 2. For every $S \subset \mathbb{N}$ we have $S \in \mathcal{D}_{\delta}$ if and only if for each representative system $\left\{a_{1}^{(n)}, \ldots, a_{k_{n}}^{(n)}\right\}$ the equality

$$
\lim _{n \rightarrow \infty} \sum_{j=1}^{k_{n}} \mathcal{X}_{S}\left(a_{j}^{(n)}\right) \Delta\left(E_{j}^{(n)}\right)=\delta^{*}(S)
$$

holds.

Full analogy of the proof of Theorem 8 leads to the following assertion.

Theorem 9. Let $\{v(n)\}$ be a sequence of elements of $[0,1]$. Then this sequence is $\delta$-continuously distributed if and only if for every continuous function $f$, defined on $[0,1]$, and for every representative system $\left\{a_{1}^{(n)}, \ldots, a_{k_{n}}^{(n)}\right\}$ of $\mathcal{E}_{n}, n \in \mathbb{N}$,

a) there exists a constant $\Phi(f)$ such that the equality

$$
\lim _{n \rightarrow \infty} \sum_{j=1}^{k_{n}} f\left(v\left(a_{j}^{(n)}\right)\right) \Delta\left(E_{j}^{(n)}\right)=\Phi(f)
$$

holds

and

b) for every real number $x$ the equality

$$
\lim _{\varepsilon \rightarrow 0^{+}} \limsup _{n \rightarrow \infty} \sum_{j=1,\left|v\left(a_{j}^{(n)}\right)-x\right|<\varepsilon}^{k_{n}} \mid f\left(v\left(a_{j}^{(n)}\right) \mid \Delta\left(E_{j}^{(n)}\right)=0\right.
$$

holds uniformly with respect to $\left\{a_{1}^{(n)}, \ldots, a_{k_{n}}^{(n)}\right\}, n \in \mathbb{N}$.

Theorem 8 implies the following result.

Corollary 5. The set of all $\delta$-continuously distributed sequences of elements of $[0,1]$ is closed with respect to the supremum norm. 


\section{References}

[1] Buck R.C. The measure theoretic approach to density. Amer. J. Math. 1946, 68 (4), 560-580. doi:10.2307/2371785

[2] Buck R.C. Generalized Asymptotic Density. Amer. J. Math. 1953, 75 (2), 335-346. doi:10.2307/2372456

[3] Drmota M., Tichy R.F. Sequences, Discrepancies and Applications. In: Lecture Notes in Mathematics. Springer, Berlin Heidelberg, 1997. doi:10.1007/BFb0093404

[4] Freedman A.R., Sember J.J. Densities and summability. Pacific J. Math. 1981, 95 (2), 293-305.

[5] Kuipers L., Niederreiter H. Uniform Distribution of Sequences. John Wiley \& Sons Inc., New York, London, Sydney Toronto, 1974.

[6] Leonetti P., Tringali S. On the notions of upper and lower density. Proc. Edinb. Math. Soc. 2020, 63 (1), 139-167. doi:10.1017/S0013091519000208

[7] Iacò M.R., Paštéka M., Tichy R.F. Measure density for set decompositions and uniform distribution. Rend. Circ. Mat. Palermo 2015, 64 (2), 323-339. doi:10.1007/s12215-015-0202-1

[8] Paštéka M. On four approaches to density. Peter Lang Publ., Bern, 2014.

[9] Paštéka M. Remarks on one type of uniform distribution. Unif. Distrib. Theory 2007, 2 (1), 79-92.

[10] Sonnenschein D.J. A general theory of asymptotic density. Doctoral dissertation, Simon Fraser University, Canada, 1978

[11] Schoenberg I. Über die asymptotische Verteilung reeler Zahlen mod 1. Math. Z. 1928, 28, 171-199. doi: 10.1007/BF01181156

[12] Strauch O., Porubský S. Distribution of Sequences: A Sampler. Peter Lang Publ., Bern, 2005.

[13] Weyl H. Über die Gleichverteilung von Zahlen mod. Eins. Math. Ann. 1916, 77, 313-352. doi:10.1007/BF01475864

Received 17.06.2020

Revised 15.09.2020

Паштека М. Замітки про неперервно розподілені послідовності // Карпатські матем. публ. — 2021. — T.13, №1. — C. 89-97.

У першій частині статті ми означаємо поняття щільності як певний тип скінченно адитивної ймовірнісної міри та функції розподілу послідовностей за цією щільністю. Потім ми виводимо деякі прості критерії, що забезпечують неперервність функції розподілу заданої послідовності. Ці критерії ми застосовуємо до послідовностей ван дер Корпута. Аоведено критерії типу Вейля неперервності функції розподілу.

Ключові слова і фрази: рівномірний розподіл, шільність, послідовність ван дер Корпута. 